\title{
A Study on the Gray Value Distribution Contour Curve Extraction Based on the Image of the Fracture Surface of the Material and Its Fractal Dimension
}

\author{
Tian $\mathrm{Ju}^{1}$, Liu Chuansheng ${ }^{1}$, Wu Chengbao ${ }^{1,2}$, Chen Zhenghua ${ }^{3}$, Liang Jizhao ${ }^{2}$, \\ Li Luyao ${ }^{1}$ \\ ${ }^{1}$ School of Aircraft Maintenance Engineering, Guangzhou Civil Aviation College, Guangzhou \\ 510430, P.R. China) \\ ${ }^{2}$ College of Mechanical and Automobile Engineering, South China University of Technology, \\ Guangzhou, 510640, P.R. China) \\ ${ }^{3}$ Maintenance Engineering Department, Guangzhou Baiyun International Airport Ground Servicing \\ LTD. Company, Guangzhou, 510470, P.R. China)
}

Keywords: Material; fracture surface; gray value distribution; contour curve; fractal dimension

\begin{abstract}
It was proposed that the gray value distribution contour curve of the fracture surface of material was used to represent the true contour curve of fracture surface, the gray value distribution contour curve was extracted by the image analysis software IPP(Image Pro-Plus), and the fractal dimension of the gray value distribution contour curve of the fracture surface was measured by the structure function method as polypropylene (PP) was the sample, in order to characterize the morphological characteristic of the fracture surface of material. The results indicated that the gray value distribution contour curve could reflect the multilevel of the fracture surface of the material; the fractal dimension of gray value distribution contour curve of PP was 1.6375, the linear degree of fitted by the structure function method was high and the correlation coefficients obtained were more than 0.99. The strong 1inear correlation indicated that impact fracture surface of the PP had significant fractal characteristics. Thus using the fractal dimensions to describe the fracture surface morphology of material may provide a new approach to investigate the morphology of fracture surface of materials.
\end{abstract}

\section{Introduction}

The morphology of tensile, impact, and bend fracture surfaces of materials were treated as the deviation of average surface in the traditional traditional analytical method, and characterized by the common statistical parameters and roughness as characterizing the other rough face [1-3], and which were used to propose the fracture mechanism of materials. But, the reports on the micro-morphology characteristics of fracture surface found that an uneven fracture surface with low smoothness would formed during subjecting to external load due to the different material structure and molding parameters, and the surface topography of the fracture had multi-levels [4-6]. The much finer structure in the fracture surface will appear constantly with the increasing of magnification times of the scanning electron microscope.

Moreover, the contour curve of the fracture surface is not smooth at different magnifications; there is no tangent at any point of the contour curve. So, the contour curve function is not differentiable; in addition, when the contour is enlarged, the probability distribution of the enlarged surface is very similar with the original surface.

Early in 1978, Sayles [7] believed that the surface morphology was unstable stochastic processes, the traditional some statistical parameters could not characterize the random behavior and detailed features of the fracture morphology comprehensively. As the fractal geometry came into being, in 1984, Mandelbrot [8] first proposed to characterizing the morphology characteristic of fracture surface of metal quantitatively by the fractal dimension; from then on, the fractal geometry was used to characterize the characterize the morphology characteristic of fracture surface of materials widely. In the last years, the Duan Chenlong [9] found that the morphology of fracture surface of 
printed circuit board and its components were fractal. The study of Xian Yu [10] took the bamboo-plastic composite as the experimental sample, obtained the impact fracture surface of the composite, and found that the fracture surfaces of the composites were fractal obviously. Wang Liang [11] analyzed the fracture morphology characteristics of the 6061 aluminum alloy composites reinforced aluminum borate, and found it was fractal.

It was one of the research highlights on characterizing the fractal characteristic of fracture surface that the relative principles of fractal geometry was used to process the contour curve of the fracture surface of materials, and the fractal dimension was used to characterize the morphology characteristic of the fracture surface. Many kinds of methods used to calculated the fractal dimension were developed based on the contour curve of fracture surface of materials, and divided into two types [12-13]: the first type was indirect method, including vertical section method, variable metric method, the secondary electron line method, and structure function method, etc; the other type was direct methods, the slit island method, box dimension method, and so on. The structure function method among the methods mentioned above was accurate, efficient, and stably. In the past study[14-15], the fractal characteristic of fracture surface of polymer composite were investigated as the samples of hollow glass bead filling the ABS polymer composite and the polypropylene filled with nano-calcium carbonate composite. In this study, the gray value distribution contour curve of fracture surface of PP material based on the SEM of the fracture was extracted, and the fractal dimension was calculated by the structure function method.

\section{Extracting the Gray Value Distribution Contour Curve}

It is well known that salient points in the fracture surface of the material are bright pixels in the SEM, while the pits in the fracture surface are the darker pixels, the slope between the salient points and pits in the fracture surface displays shade with different gray value. so, the smoothness degree, coarseness degree, and the difference among the morphology characteristics of different fracture surface of material can be judged by observing the bright and shade degree of the SEM. But, this method can only characterize the morphology characteristic quantitatively. If extracting the gray value of the pixel point in the SEM, the relative position of the mass point to average surface of the fracture surface can be reflected; if obtaining gray value of a series of continuous point, one can get a continuous contour curve of gray value, the contour curve can reflect the relative positions of all mass points relative to the average surface of the fracture surface.

The idea mentioned above can be realized by the digital technique of picture with the development of image processing and analyzing technique. In this study, a image processing software named IPP (Image Pro-Plus) was used to extract gray value of the SEM and the gray value distribution contour curve based on the SEM of the fracture surface of the material. The specific operations are as following:

1) To open the IPP, and import the SEM of the fracture surface of the material;

2) To use the pixel obtaining tool to obtain the gray values of an optional horizontal line in the graphical map of the SEM;

3) To import the gray values to the excel;

4) To import the gray values in the Excel into Matlab 7.1, and draw the gray values, then the gray value distribution contour curve is obtained .

\section{Results and Discussion}

Gray Value Distribution Contour Curve of the Fracture Surface of PP Material. Figure 1 shows the 5 optional and horizontal positions in the SEM of the PP. One can see from Figure 1 that the fracture surface is uneven, looks like the wave. If magnifying the fracture surface, much finer structure like little wave will be displayed. 


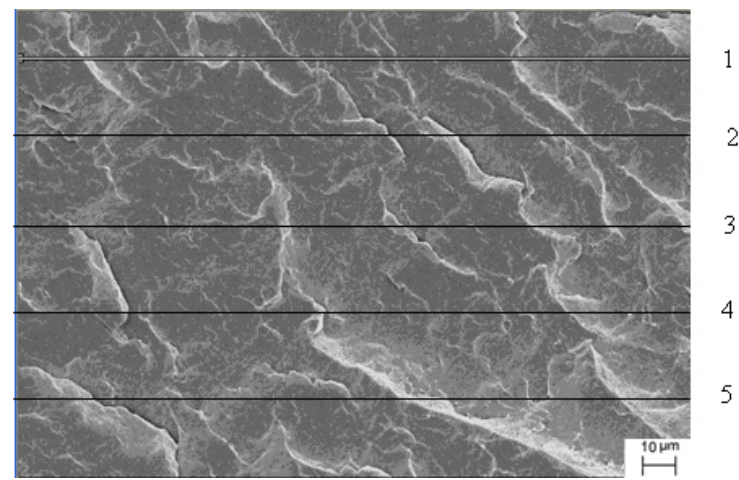

Fig.1 five horizontal lines of the image of the fracture surface

The gray valves of at different pixel point of the horizontal lines of the SEM of the fracture surface was gain based on the extraction method mentioned above. The gray value distribution contour curve of the fracture surface of PP material were showed from Figure 2 to Figure 6.

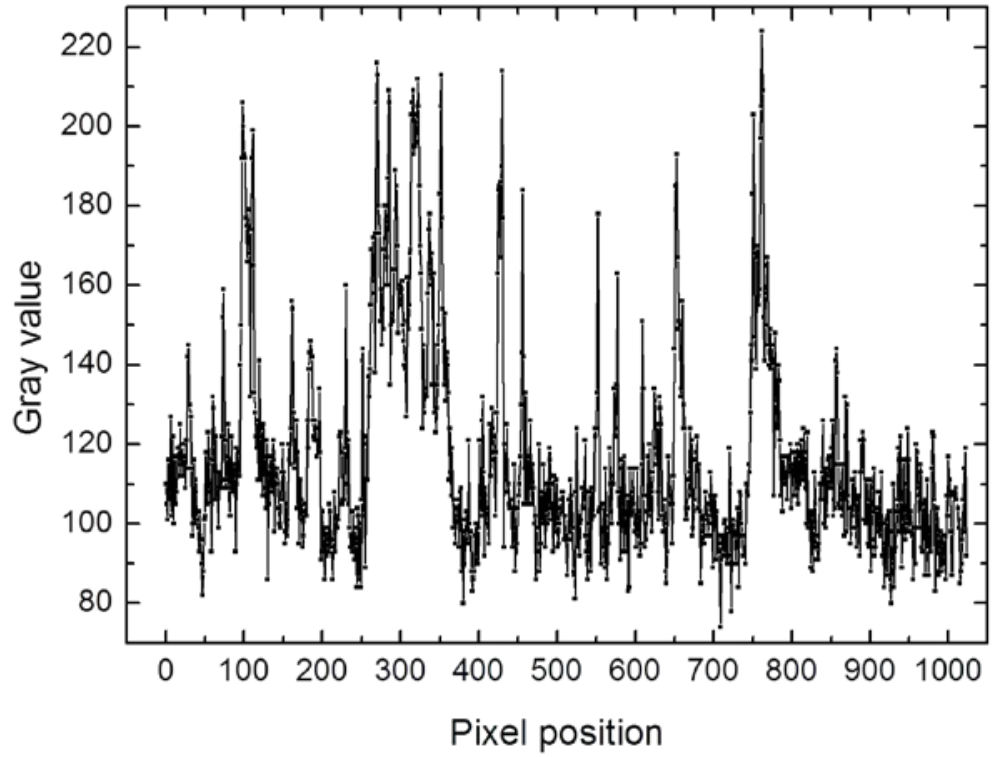

Fig.2 Gray value distribution contour curve of the horizontal line 1

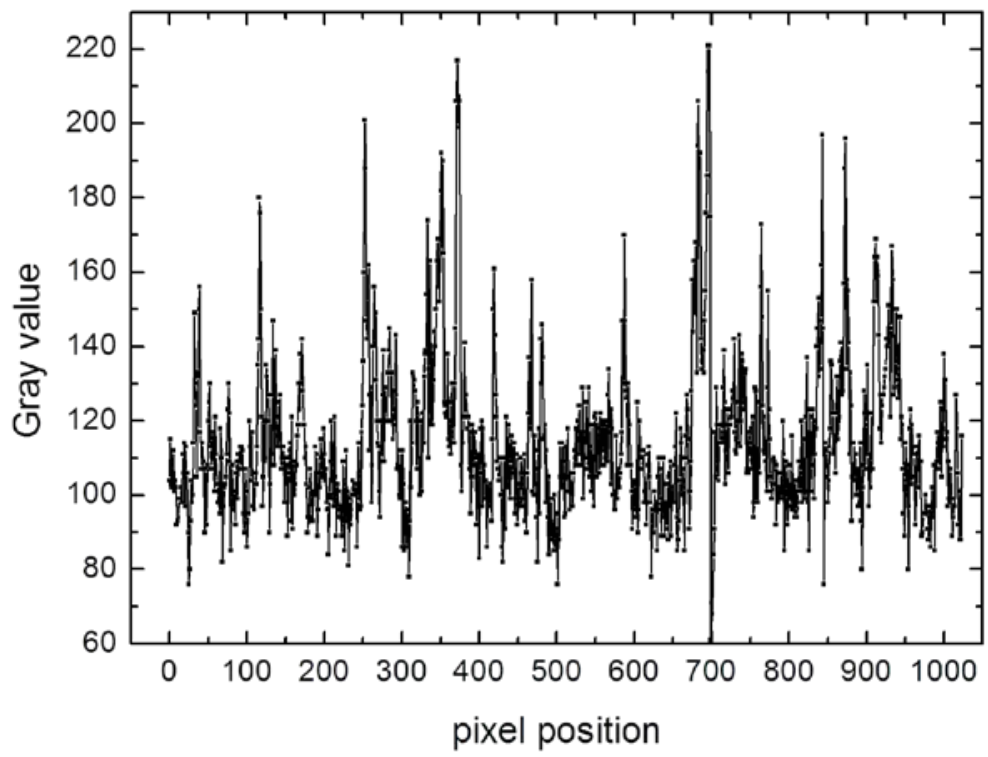

Fig.3Gray value distribution contour curve of the horizontal line 2 


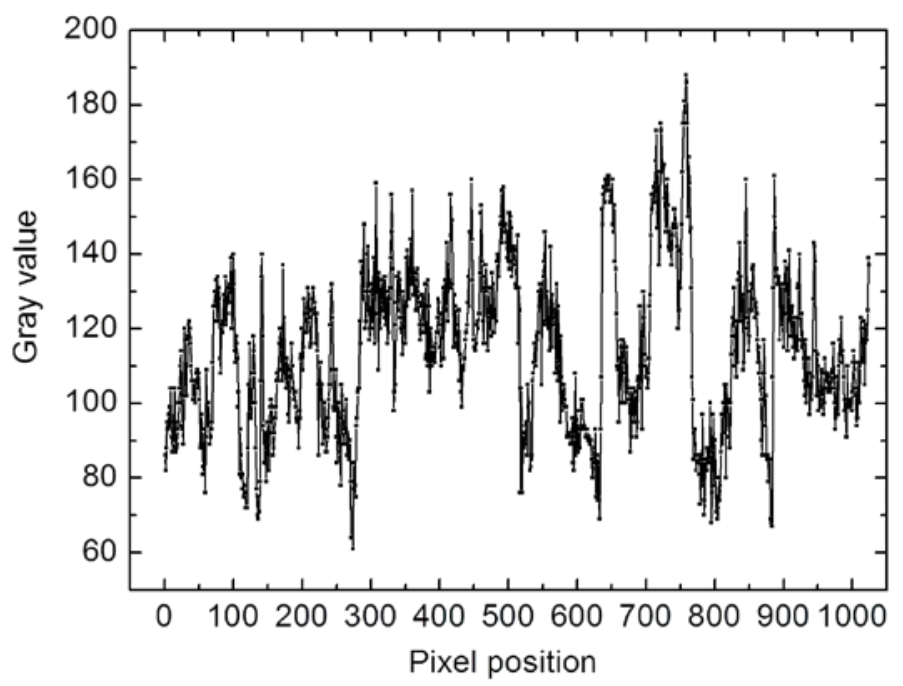

Fig.4 Gray value distribution contour curve of the horizontal line 3

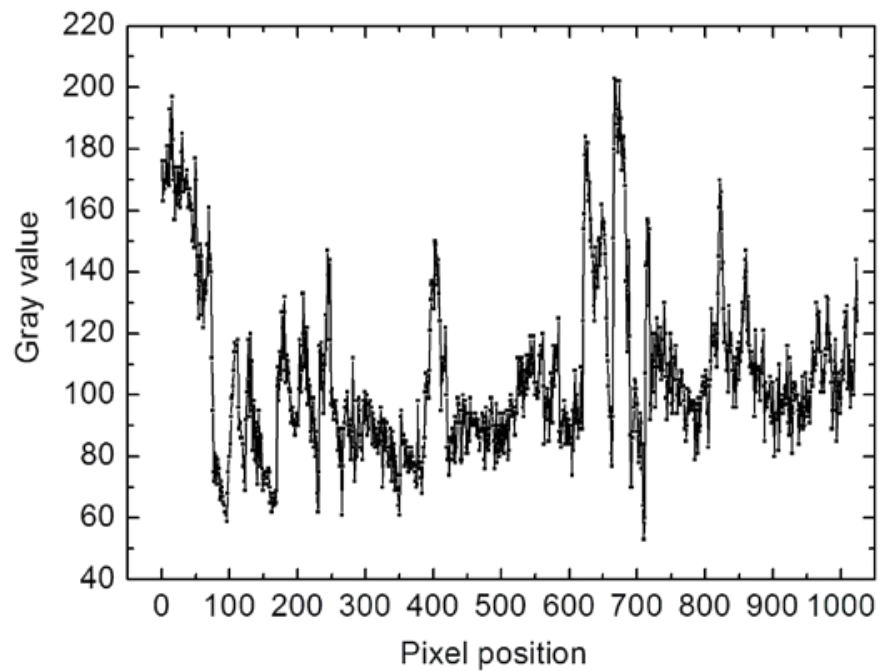

Fig.5Gray value distribution contour curve of the horizontal line 4

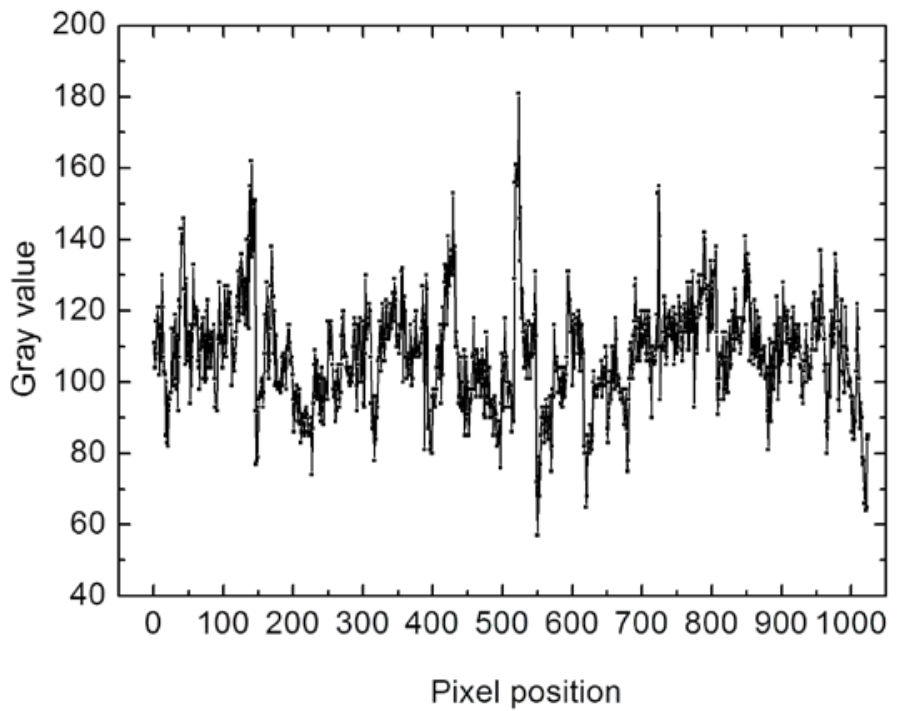

Fig.6 Gray value distribution contour curve of the horizontal line 5

From Figure 2 to Figure 6, we can find that the gray value distribution contour curves at the different positions of the image of the fracture surface of the pure PP sample have the similar 
profiles and have the similar gradation level. That is to say, the gray value distribution contour curve can reflect the true morphology of the fracture surface of the PP.

Fractal Dimension of the Gray Value Distribution Contour Curve. The theory principle of the structure function as following: [16 17]

$$
S(t)=\left\langle[Z(x+t)-Z(x)]^{2}\right\rangle=c t^{4-2 D_{L}}
$$

Where, $Z(x)$ is gray value; $x$ is the pixel position; $S(t)$ is arithmetic mean value of the difference quadratic; $t$ is optional value of the time interval, and is integer; $D_{L}$ is fractal dimension of the contour curve. The $S(t)$ is calculated at the different $t$ based on the discrete data of the contour curve.

To take the logarithm of Eq.1, and Eq.2 is got:

$$
\lg [S(t)]=\lg c+\left(4-2 D_{L}\right) \lg t
$$

To fit the $\lg [S(t)]_{\text {and }} \lg t$ with least square method one can get a equation of linear regression:

$$
Y=C+D X
$$

Where $Y=\lg [S(t)], C=\lg c, \quad X=\lg t$.

The slope of the fitting equation $\mathrm{D}$ :

$$
D=4-2 D_{L}
$$

Then the Equ.4 can be transferred to:

$$
D_{L}=\frac{4-D}{2}
$$

The DLs of five different horizontal lines of SEM of the PP was calculated, and the average of the five DLs was regarded as the final result. The calculated curves and fitting curves for DL of fracture surface were shown in Figure 7.

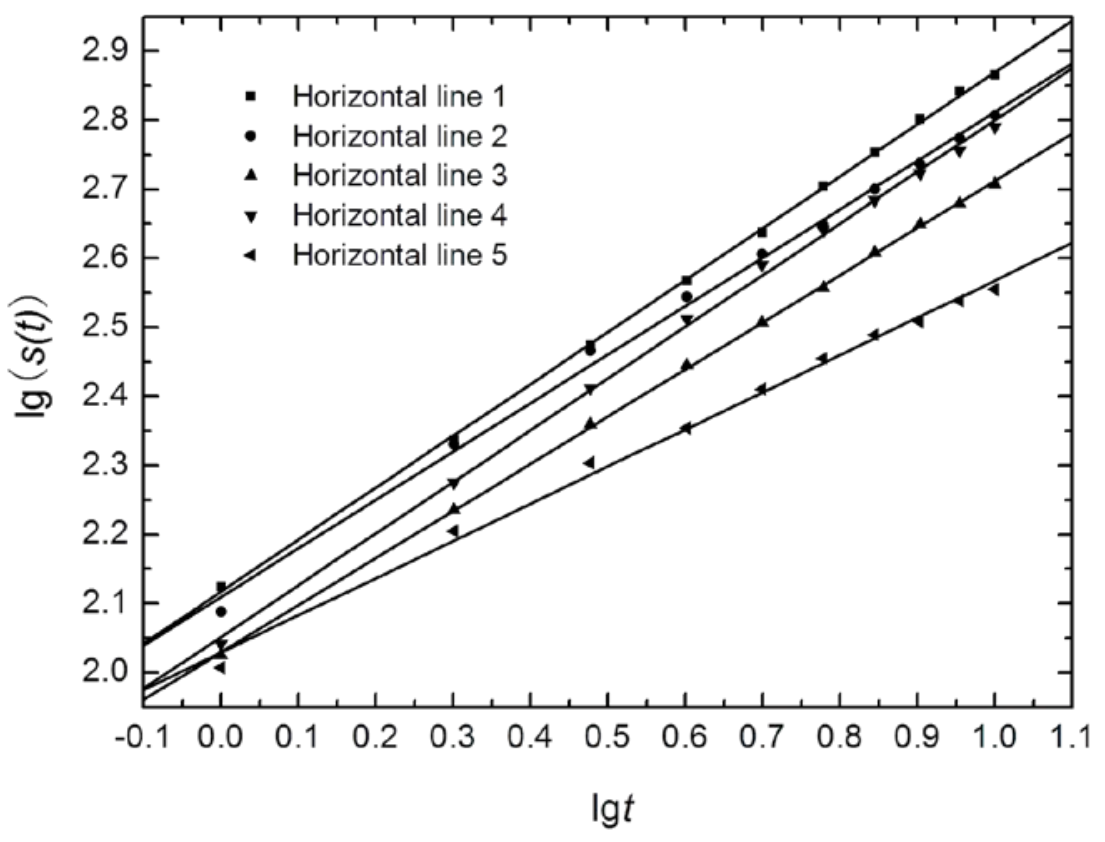

Fig.7calculated curve for $D_{L}$ value of fracture surface

It can be seen from Figure 7 that the fitting curve between $\operatorname{lgs}(t)$ and $\lg (t)$ of gray value distribution contour curve of fracture surface at the different horizontal position of the SEM is accordance with linear model:

$$
\lg (S(t))=b+\alpha \lg t
$$

Where $a$ and $b$ are constants related to the gray value distribution, the larger the $a$ is, the larger the $D_{L}$ is, the more complex the contour curve is, and the more fine structure is. From Eq. 5, 
one can know that $D_{L}=2-a / 2$, the $\alpha, b, R$ and $D_{L}$ related to the calculation of $D_{\mathrm{L}}$ are shown in Table 1.

Table $1 D_{L}$ value of fracture surface

\begin{tabular}{ccccc}
\hline Horizontal line & $\begin{array}{c}\text { Linear regression } \\
\text { equations }\end{array}$ & $R$ & $D_{L}$ & $\begin{array}{c}\text { The average } \\
D_{L}\end{array}$ \\
\hline 1 & $\lg (s(t))$ & 0.999 & 1.623 & \\
& $=2.1175+0.7524 \lg t$ & 8 & 8 & \\
2 & $\lg (s(t))$ & 0.998 & 1.648 & \\
& $=2.1099+0.7021 \lg t$ & 5 & 9 & \\
3 & $\lg (s(t))$ & 0.999 & 1.658 & 1.6375 \\
& $=2.0299+0.6827 \lg t$ & 8 & 7 & \\
4 & $\lg (s(t))$ & 0.999 & 1.625 & \\
5 & $=2.0520+0.7488 \lg t$ & 2 & 6 & \\
& $\lg (s(t))$ & 0.997 & 1.630 & \\
& $=2.0292+0.5391 \lg t$ & 6 & 4 & \\
\hline
\end{tabular}

We can see from Table 1 that the correlation coefficient of linear regression analysis is larger than 0.99. The high correlation indicates that the contour curve of fracture surface is fractal evidently.

\section{Conclusions}

The gray value distribution contour curve of fracture surface of material was extracted by the image processing software and the data processing software, and the fractal dimension of the ray value distribution contour curve of fracture surface was calculated by the structure function method based on the relationship between the morphology characteristic of the fracture surface of the material and its pixel gray value distribution contour curve. The gray value distribution contour curve based on the SEM of the fracture surface of the material could reflect the true morphology characteristic of the fracture surface. The confidence coefficient of structure function method used to calculate the fractal dimension of the gray value distribution contour curve is high. So, the fractal dimension could be used to characterize the multilevel characteristic of the fracture surface of the material quantitatively.

\section{Acknowledgements}

This work is supported by the The civil aviation scientific and technological innovation guiding capital.

\section{Reference}

[1] Yao Changfeng,Wu Daoxia, Jin Qichao, Huang Xinchun,Ren Junxue, Zhang Dinghua. Influence of high-speed milling parameter on 3D surface topography and fatigue behavior of TB6 titanium alloy[J]. Transactions of Nonferrous Metals Society of China, 2013, 23: 650-660.

[2] Yu Bin, Jin Qingchen. Quantitative Measurement and Analysis of Macroscopical Morphology of Fracture Sureface[J]. Nonferrous Metals, 2010, 62(3): 1-5.

[3] Liu Zhidong, Yu Bin. Quantitative Study of Macroscopical Metallic Fracture Surface Roughness[J]. Aerospace Materials \& Technology, 2009,39(5): 20-23.

[4] Liu Shuhao, Chen Bing, Yang Jing. The Study of Strip Shearing Section Level Distribution of Shearing Process[J]. Machinery Design \& Manufacture, 2012(10):108-110.

[5] Liang J.Z., Li R.K.Y.. Mechanical properties and morphology of galss bead-filled polypropylene 
[J]. Polymer Composites, 1998, 19(6): 698-703.

[6] Gokhale A.M., Underwood E.E.. A general method for estimation of fracture surface roughness: part I. theoretical aspects [J]. Metallurgical Transactions A, 1990, 21A: 1993-1999.

[7] Sayles R. S., Thomas T. R.. Surface topography as a non-stationary random process [J]. Nature, 1978, 271: 431-434.

[8] Mandelbrot B.B., Passoja D.E., Paulla A.J.. Fractal character of fracture surface of metals [J]. Nature, 1984(19): 721-722.

[9] Duan Chenlong, Zhao Yuemin, Tang Ligang, He Yaqun, Song Shulei. Fractal Characterization of Material Fracture of Waste Printed Circuit Boards[J]. Journal of Central South University (Science and Technology), 2009, 40(1): 78-82.

[10] Xian Yu, Wang Cuicui, Wang Ge, Cheng Haitao. Fractal Characterization of Impact Strength Fracture of Bamboo Plastic Composites with Core-shell Structure[J]. Transactions of the Chinese Society of Agricultural Engineering, 2015, 31(12): 295-260.

[11] Wang Liang, Wang Lidong, Fei Weidong. Fractal analysis of fracture surfaces in aluminum borate whisker-reinforced aluminum alloy 6061 composite [J]. Transactions of Nonferrous Metals Society of China, 2011( 21): 461-466.

[12] Wu Chengbao, Liang Jizhao. Fractal Characteristics and Determining Methods of Fracture of Inorganic Grain Filled Polymer Composites [J]. Plastics Science and Technology, 2007, 35(8): 60-64.

[13] Liu Anzhong, Liu Yihua, Wang Jionghua. A Research on the Relationship Between the Static Friction Conefficients of Fractures and Their Fractal Dimensions For $\mathrm{TiO}_{2} / \mathrm{ABS}$ Grain Composite Materials [J]. Journal of Anhui Institute of Architecture \& Industry, 2003, 11(1): 69-71.

[14] Liang Jizhao, Wu Chengbao. Relationship between the Fractal Dimension of Fracture Surface and the Impact Strength of ABS/HGB Composites[J]. Materials Science and Engineering, 2010, (2): 178-182.

[15] Liang Jizhao, Wu Chengbao. Study on Relationship Between Impact Strength and Fracture Surface Fractal Dimension of PP/Nano-CaCO ${ }_{3}$ Composites[J]. Journal of Materials Engineering, 2009,(10):53-56.

[16] Li Chao, Zhang Jing, Jiao Yu, Zhao Man. Research on the Relationship of Different Surfaces Roughness and Fractal Dimensions of PEEK[J]. 2011,36(2): 17-21.

[17] S Oudjemia, A Zaylaa, S Haddab, JM Girault. Coarse-grained multifractality analysis based on structure function measurements to discriminate healthy from distressed foetuses [J]. Computational and Mathematical Methods in Medicine, 2013(1):152828-152828. 